\title{
Geociênceiajas
}

\section{As propriedades friccionais de areias de quartzo (natural e colorida): medidas efetuadas em experimentos físico-analógicos e em um ring-shear tester}

\author{
The frictional properties of quartz sands (natural \\ and colored): measurements from analogue \\ experiments and from a ring-shear tester
}

Caroline Janette Souza Gomes

Professora Associada III, Departamento

de Geologia, Escola de Minas,

Universidade Federal Ouro Preto

caroline@degeo.ufop.br

Jefter Natan de Moraes Caldeira

Bolsista de Iniciação Científica (PIBIC),

Departamento de Geologia, Escola de Minas,

Universidade Federal Ouro Preto

jefternatan@hotmail.com

\section{Resumo}

Com o intuito de se determinarem as propriedades friccionais da areia de quartzo empregada em modelagens físico-analógicas foram desenvolvidos dois ensaios de laboratório. No primeiro, estimou-se o coeficiente de atrito interno a partir do ângulo de mergulho de falhas de empurrão, em caixas de areia, e, no segundo, realizaram-se medições em um ring-shear tester. Nesse aparelho, determinaram-se as propriedades, separadamente, para areias de quartzo tingidas em diferentes cores, da tinta Acrilex. Os valores do coeficiente de atrito interno revelaram-se mais altos e mais preciso no ring-shear tester do que nos modelos analógicos. A comparação dos resultados com dados da literatura mostrou que as areias testadas se enquadram em uma faixa de valores bastante elevada. Além disto, o estudo no ring-shear tester demonstrou que as areias tingidas em diversas cores possuem propriedades friccionais distintos da areia incolor (ângulo de atrito interno $=42,7^{\circ}$ ) e entre si. O fato é atribuído a diferenças na composição química da tinta e deve ser avaliado com cuidado em futuros experimentos, em caixas de areia.

Palavras-chave: Coeficiente de atrito interno; areia de quartzo; modelos físicoanalógicos, ring-shear tester.

\begin{abstract}
To improve knowledge of the quartz sand used in analogue modeling, two laboratory tests were carried out to investigate the frictional properties of colored sand. The first one estimated the coefficient of internal friction from the reverse fault dip simulated in a pack of different colored dry sand layers. The second test consisted of measuring stress-strain curves separately for different colored sand using a ring-shear tester. The coefficient of internal friction determined from fault dips is significantly lower and less precise than that determined on the same material with a ring-shear tester. Comparisons with previous granular material tests reveal that although our ring-shear test results are high, they are within the range of values reported in literature. Our results also show that natural and colored sands have different angles of internal friction, and that the Acrilex dye produces a distinct mechanical behaviour for different colored sand. The variation is related to the chemical composition of sand dyes and should be carefully evaluated in future sandbox experiments.
\end{abstract}

Keywords: Coefficient of internal friction; quartz sand; analogue experiments; ringshear tester. 


\section{Introdução}

Experimentos físico-analógicos, em escala, têm sido usados para o estudo dos mecanismos da deformação e da geometria das estruturas tectônicas. O produto mais comumente utilizado para simular a deformação da crosta superior é a areia de quartzo, de baixa coesão. Estudos recentes têm demonstrado que a tese de que a crosta rúptil e a areia de quartzo, rotineiramente empregada para simular a deformação rúptil, possuam propriedades friccionais constantes e se deformem segundo o critério de ruptura de Cou- lomb (Byerlee, 1978) (Figura 1A) constitui uma simplificação. Lohrmann et al. (2003) demonstram que tanto as rochas naturais quanto os materiais granulares, utilizados na modelagem físico-analógica, são caracterizados por um comportamento inicialmente elástico seguido por um estágio de strain-hardening, plástico, até ocorrer ruptura. Com a deformação progressiva inicia-se o movimento sobre a superfície da falha, caracterizada por strain softening, com a tensão cisalhante decrescendo até alcançar valores estáveis

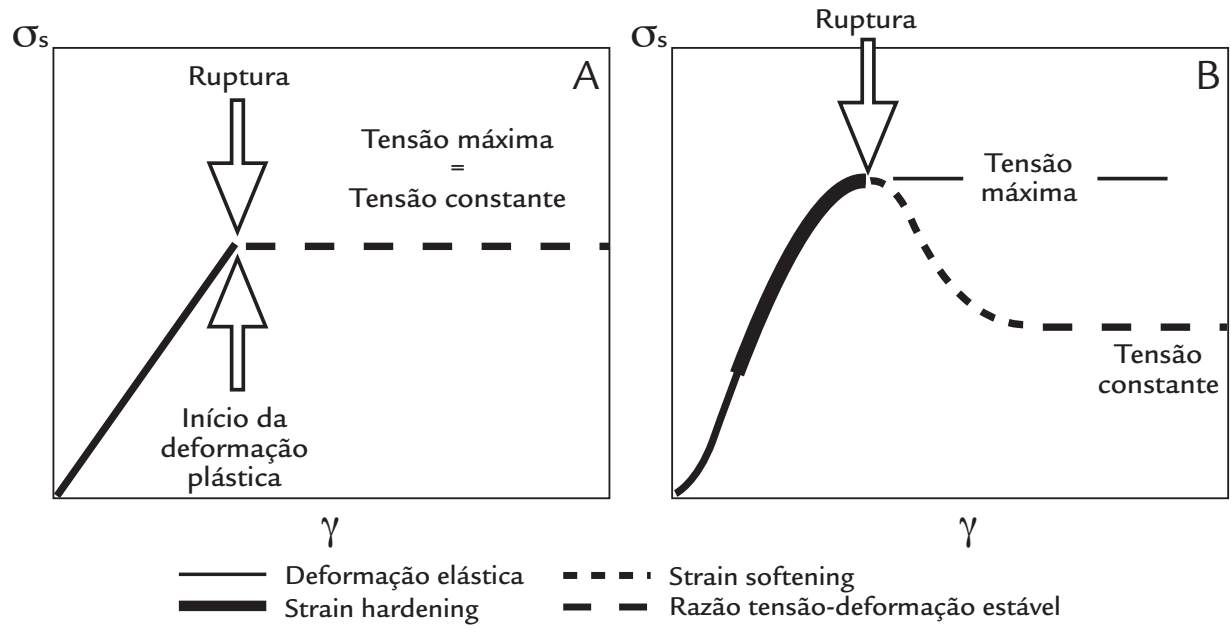

O coeficiente de atrito interno reflete a maneira como os grãos deslizam entre si, enquanto a coesão é a medida da tensão cisalhante à qual o material resiste, na ausência de qualquer tensão normal confinante (Mandl, 1988). Na modelagem física analógica, utilizam-se três métodos para a determinação das propriedades friccionais dos materiais granulares. Todos estes se baseiam no critério de ruptura de Coulomb, conhecido pela equação:

$\sigma_{\mathrm{s}}=\mathrm{C}+\mu \sigma_{\mathrm{N}}$,

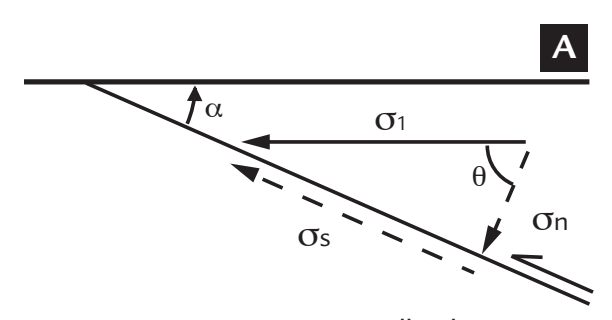

Falha de empurrão

O segundo método foi desenvolvido por Hubbert (1951). O autor desenvolveu um aparato, denominado shearbox, onde o material a ser estudado é submetido a tensões normais de carregamento, crescentes, até ocorrer a rup- em que $\sigma_{s}$ corresponde à tensão cisalhante limite, $\mathrm{C}$, à coesão do material, $\mu$, ao coeficiente de atrito interno e $\sigma_{\mathrm{N}}$, à tensão normal.

O método mais simples para a determinação da tensão cisalhante crítica do material granular, sob baixas condições de tensões normais, como as que agem sobre os modelos físicos, estima o coeficiente de atrito interno $(\mu)$ a partir do ângulo de mergulho de planos de falhas (Krantz, 1991). A Figura 2A mostra que, para falhas de empurrão, tem-se:

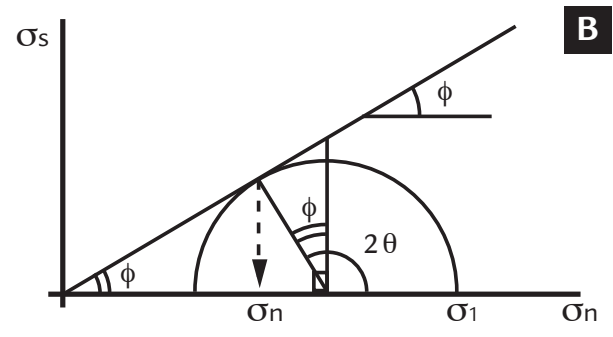

tura. Este é o princípio básico também dos shear-testers rotacionais utilizados na mecânica dos solos, desde a década de 30, e, nos quais, Schulze (1994) se baseou, para a construção de um ringshear tester, aqui utilizado.
(Figura 1B).

Os referidos autores argumentam que, apesar de a "reologia Coulomb" constituir uma simplificação, ela pode ser adotada para descrever o comportamento mecânico das rochas naturais, em escala regional. Para o caso dos materiais granulares, no entanto, é necessário determinarem-se as propriedades friccionais, o coeficiente de atrito interno (e o ângulo de atrito interno) e a coesão, nos dois estágios da deformação, de ruptura e de tensão cisalhante constante.

Figura 1

Diagramas de tensão cisalhante $\left(\sigma_{\mathrm{s}}\right)$

versus deformação $(\gamma)$ para

(A) materiais com comportamento de Coulomb ideal.

(B) rochas naturais e materiais analógicos granulares empregados na modelagem física analógica (modificado de Lohrmann et al., 2003).

$\theta=90^{\circ}-\alpha$,

onde $\theta$ é o ângulo entre o vetor tensão principal máxima, $\sigma_{1}$ e a componente tensão normal, e $\alpha$ corresponde ao ângulo de mergulho da falha. Sabendo-se que, $\mu=\operatorname{tg} \Phi$

e, pela Figura $2 \mathrm{~B}$,

$\Phi=2 \theta-90^{\circ}$

então:

$\mu=\operatorname{tg}\left(2 \theta-90^{\circ}\right)$

onde $\mu$ corresponde ao coeficiente de atrito interno e $\Phi$, ao ângulo de atrito interno (a declividade da reta).

Figura 2

(A) Esquema de uma falha de empurrão com a indicação do ângulo de mergulho da falha, $\alpha$, e do ângulo $\theta$.

(B) Diagrama de Mohr, com a representação dos ângulos de atrito interno, $\Phi$, e $2 \theta$. Observar que $2 \theta-90=\Phi$.

Para os fins da modelagem física, o ring-shear tester constitui o terceiro método. Trata-se de um aparelho que trabalha com baixas magnitudes de tensões, comparáveis com aquelas aplicadas aos pacotes de areia, nas caixas 
de experimentos.

Nesse aparelho, um software registra os resultados, tanto da tensão cisalhante crítica quanto da tensão estável e, quando solicitada, também da tensão de reativação da falha, sob várias condições de tensão normal de carregamento.

No presente trabalho, apresenta-se o primeiro estudo desenvolvido no ringshear tester RST-XS, recentemente adquirido pelo Laboratório de Modelagem Tectônica do Departamento de Geologia

\section{Experimentos analógicos}

\section{Materiais e métodos}

Os experimentos foram montados com areia de quartzo seca, tingida com tinta de tecido Acrilex, nas cores vermelha, azul e amarela (daqui em diante, referidas como diferentes "tipos de areia" ou simplesmente "diferentes areias"). Antes do tingimento, a areia lavada foi peneirada até a faixa granulométrica muito fina, de $210 \mu \mathrm{m}$ a $350 \mu \mathrm{m}$. No entanto, duas das três tintas empregadas, a azul e a amarela, causaram um ligeiro aumento de granulometria da areia, decorrente da película de tinta que se formou em torno dos grãos de areia. Curiosamente, a tinta vermelha não causou o mesmo efeito.

Foram utilizadas caixas de acrílico, com espaço interno de $20 \mathrm{~cm}$ x $20 \mathrm{~cm}$ (Figura 3), com paredes laterais transparentes, o que permite a visualização da deformação progressiva. Uma parede móvel é acoplada a um motor que gera a compressão do pacote de areia, a uma velocidade constante, comumente usada para a simulação da deformação rúptil, de $2 \mathrm{~cm} / \mathrm{h}$.

Figura 3

(A) Bloco-diagrama da caixa de experimento com a posição dos cortes. A seta indica o sentido da compressão.

(B) Esboço do experimento, em perfil, com a representação dos segmentos $\mathrm{Xe} Y$, para a determinação do ângulo de mergulho da falha de empurrão.

\section{Resultados}

O gráfico da Figura 4 mostra o ângulo de mergulho da falha de empurrão da UFOP. O estudo tem o intuito de determinar as propriedades friccionais $\mathrm{da}$ areia incolor e da areia colorida artificialmente, com tinta Acrilex, usada no laboratório, e comparar os resultados obtidos no aparelho com aqueles determinados em modelos físico-analógicos.

Nos modelos analógicos, mediu-se o ângulo de mergulho $(\alpha)$ de falhas de empurrão geradas, por compressão, em pacotes multicamadas de areia colorida. $\mathrm{O}$ coeficiente de atrito interno $(\mu)$ e o ângulo de atito interno $(\Phi)$ foram determinados a partir das equações (4) e (5), respectivamente.

No ring-shear tester, analisaramse as areias tingidas nas mesmas cores usadas na modelagem experimental (vermelha, azul e amarela) assim como a areia incolor. Ao final dessa etapa, os resultados de cada areia colorida foram comparados com aqueles de uma mistura das três areias coloridas e com os da areia incolor.
Nas caixas de experimentos, montaram-se delgadas camadas $(\sim 2 \mathrm{~mm})$ de areia de diferentes cores até a altura de $4 \mathrm{~cm}$, utilizando-se, para essa finalidade, uma peneira de $350 \mu \mathrm{m}$. Com o intuito de se analisar o efeito de diferentes processos de montagem dos experimentos sobre o ângulo de mergulho das falhas de empurrão, foram desenvolvidas duas séries de experimentos. Em uma, a deposição da areia foi efetuada apenas por peneiramento e, na outra, cada camada recém-depositada foi levemente compactada por suaves batidas, efetuadas com uma colher. Sem a intenção de se analisarem maiores detalhes desse procedimento, mas apenas para se distinguirem as duas séries, estas foram denominadas de "não-compactadas" e "compactadas", respectivamente. Cada série era constituída por um experimento, repetido duas vezes, para minimizar erros de medidas.

$\mathrm{Na}$ caixa de experimento, gerou-se o encurtamento até o aparecimento do traço da primeira falha, em planta. Nos modelos "não compactados", o traço era visualizado após $22 \%$ de encurtamento (44 mm), e, nos "compactados", após um encurtamento menor, de 13\% (26 mm). Efetuaram-se, então, as medidas do ângulo de mergulho da falha de empurrão, em perfil. Estas foram realizadas através do vidro da parede lateral da caixa de experimentos, após se molhar o pacote de areia com água, em sucessivos cortes internos (Figura 3A). O procedimento visava tanto a determinar o ângulo de mergulho da falha em cortes, livres do atrito lateral da parede de vidro, quanto a uma análise da possível variação do ângulo de mergulho, em 3D.

O procedimento para a medição dos ângulos de mergulho da falha de empurrão seguiu a metodologia descrita por Krantz (1991) (Figura 3B): encostouse, na lateral do experimento, uma folha de transparência e, sobre esta, desenhouse um triângulo, tendo o traço da falha como hipotenusa. Considerando-se $\alpha$ o ângulo de mergulho da falha, tem-se: $\mathrm{Y} / \mathrm{X}=\operatorname{tg} \alpha, \log \alpha=\operatorname{arctg} \mathrm{Y} / \mathrm{X}$.
(A)

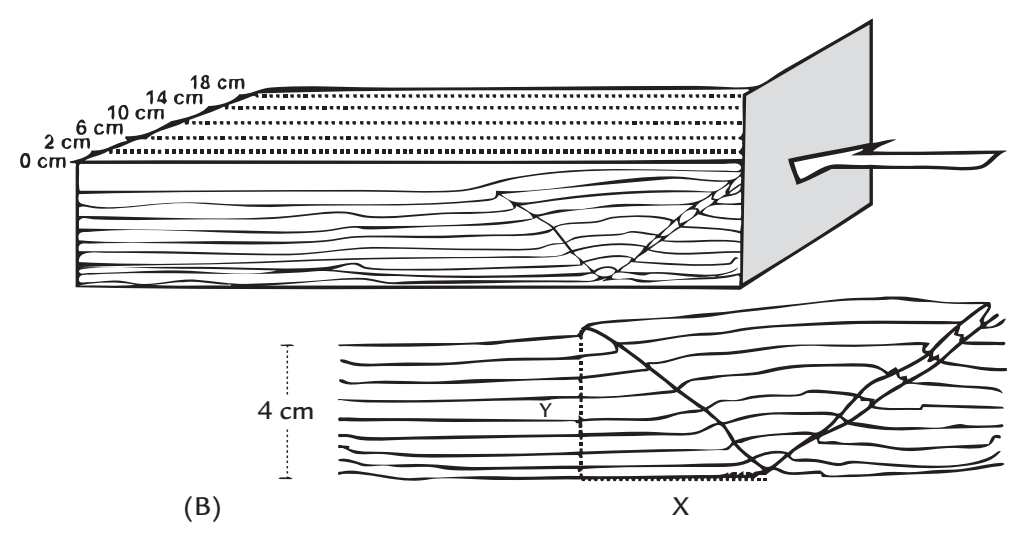

( $\alpha$ ) nas várias seções dos experimentos e a Tabela 1 apresenta, além dos ângulos de mergulho $(\alpha)$, os coeficientes de atrito $(\mu)$ e os ângulos de atrito interno $(\Phi)$, 


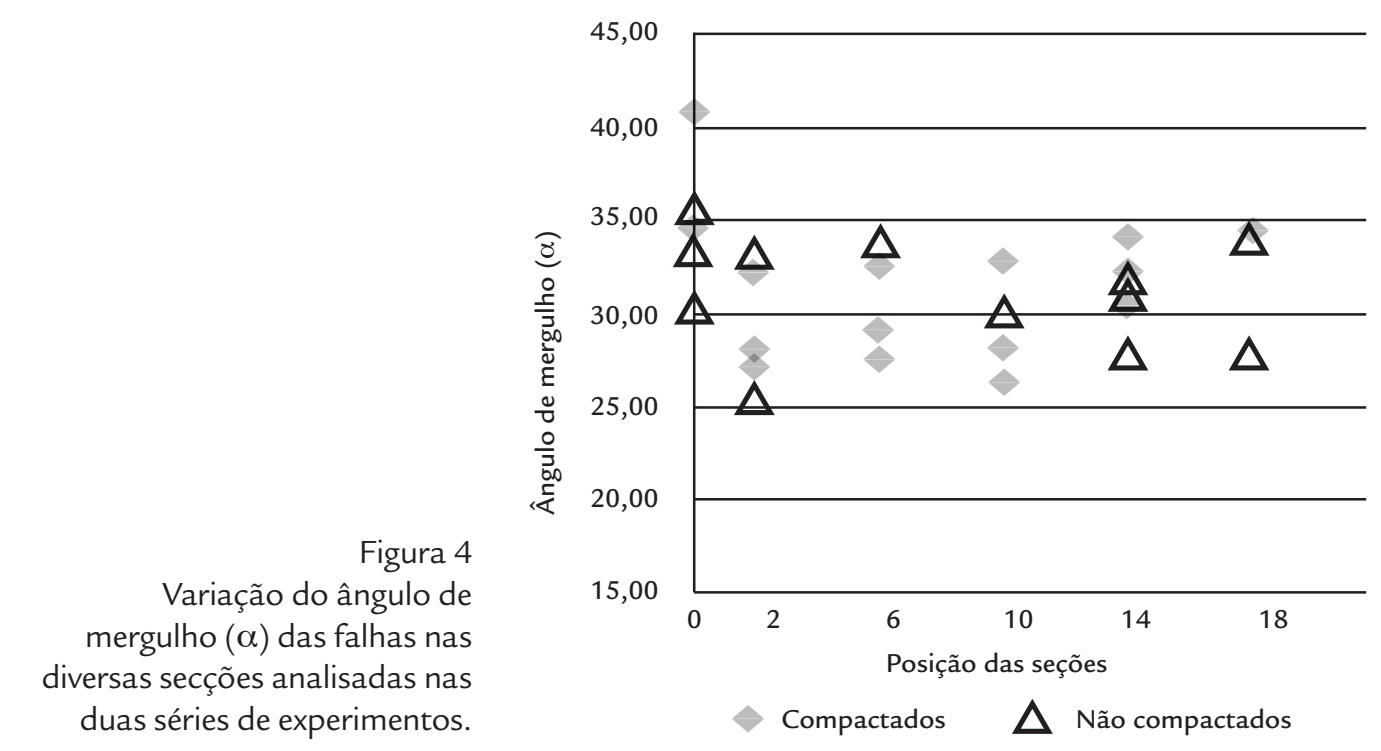

calculados a partir das equações (2), (3) e (4). A análise dos dados, na Figura 4, revela, para todos os experimentos, valores anômalos na seção zero, que constitui a parede de vidro da caixa de experimento. Nessa seção, o ângulo de mergulho $(\alpha)$ da falha de empurrão chega, na série "compactada", a 40,8 $8^{\circ}$. Valores anômalos, na parede de vidro, já eram esperados e são atribuídos ao atrito lateral. Para não influenciar os resultados finais, esses valores serão omitidos na análise, a seguir.

A Tabela 1 mostra que, nos experi-

Tabela 1

Variação de $\alpha, \Phi$ e $\mu$ (respectivamente, ângulo de mergulho das falhas, ângulo de atrito interno e coeficiente de atrito interno) encontrada nas diversas secções analisadas. A secção central dos modelos $(10 \mathrm{~cm})$ é mostrada em separado.

\section{Experimentos no ring-shear tester}

\section{Materiais e métodos}

No ring-shear tester RST-XS foram determinadas as propriedades friccionais das mesmas areias de quartzo, colorida e incolor, de granulometria entre $210 \mu \mathrm{m}$ $350 \mu \mathrm{m}$, mediante uso do software RSTCONTROL 95. Além disto, foram gerados dois gráficos, um da tensão cisalhante crítica versus tempo e outro da tensão cisalhante versus tensão normal (o diagrama de Mohr). Nesse último, os dados definem uma reta obtida a partir da análise de regressão linear simples e que constitui o critério de ruptura de Coulomb (equação (1)). Esta permite derivar o coeficiente mentos "compactados" e "não compactados" o ângulo de mergulho $(\alpha)$ da falha de empurrão apresenta valores máximos e mínimos de $\alpha$ muito próximos $\left(26,6^{\circ}\right.$ a $34,4^{\circ}$ nos "compactados" e de $25,5^{\circ}$ a $34,1^{\circ}$ nos "não-compactados"), no entanto o intervalo de variação é grande. $\mathrm{O}$ conjunto das seções mostra que, em 3D, anão varia de forma regular, em nenhuma das duas séries e que apenas os experimentos "não-compactados" apresentam, na seção central, um ângulo de mergulho praticamente constante (de $30,0^{\circ}$ a $\left.30,3^{\circ}\right)$. Ressalta-se que os cálculos efetuados, para o ângulo de atrito interno $(\Phi)$ (Tabela 1), revelam uma diferença de $0,8^{\circ}(2,75 \%)$ entre os valores mínimos das duas séries e de $2,1^{\circ}(5,38 \%)$ entre os máximos.

Esses dados mostram que as duas formas de montagem dos experimentos, "compactada" e "não compactada", influenciam a magnitude do encurtamento para o instante do falhamento, mas não modificam os ângulos de mergulho das falhas, de forma expressiva.

\begin{tabular}{c|c|c|c}
\cline { 3 - 3 } \multicolumn{2}{c|}{} & $\begin{array}{c}\text { Intervalo completo } \\
\text { dos dados }\end{array}$ & $\begin{array}{c}\text { Intervalo dos dados } \\
\text { na seção de } 10 \mathrm{~cm}\end{array}$ \\
\hline \multirow{2}{*}{$\alpha$} & s. compactada & $26,6^{\circ}$ a $34,4^{\circ}$ & $26,6^{\circ}$ a $32,9^{\circ}$ \\
\cline { 2 - 4 } & s. não-compactada & $25,5^{\circ}$ a $34,1^{\circ}$ & $30,0^{\circ}$ a $30,3^{\circ}$ \\
\hline \multirow{2}{*}{$\mu$} & s. compactada & 0,39 a 0,75 & 0,45 a 0,75 \\
\cline { 2 - 4 } & s. não-compactada & 0,40 a 0,81 & 0,57 a 0,58 \\
\hline \multirow{2}{*}{$\Phi$} & s. compactada & $21,2^{\circ}$ a $36,9^{\circ}$ & $24,1^{\circ}$ a $36,9^{\circ}$ \\
\cline { 2 - 4 } & s. não-compactada & $21,8^{\circ}$ a $39,0^{\circ}$ & $29,5^{\circ}$ a $30,0^{\circ}$ \\
\hline
\end{tabular}

de atrito interno (e o ângulo de atrito interno) assim como a coesão do material.

A metodologia de trabalho no ringshear tester seguiu aquela descrita em Ellis et al. (2004) e Panien et al. (2006). A areia é depositada em um compartimento anelar, de $30 \mathrm{ml}$, por meio de uma peneira, de $350 \mu \mathrm{m}$ de abertura, de uma altura de $7 \mathrm{~cm}$. Durante os testes, a base do compartimento é rotacionada, a uma velocidade angular constante, enquanto a tampa é mantida fixa.

Para cada tipo de areia (azul, amarela, vermelha, incolor e uma mistura na mesma proporção das três primeiras, daqui em diante denominada "areia mista"), efetuaram-se testes com cinco tensões normais de carregamento diferentes: de $800 \mathrm{~Pa}, 1200 \mathrm{~Pa}, 1600 \mathrm{~Pa}, 2000 \mathrm{~Pa}$ e $2400 \mathrm{~Pa}$ e, para assegurar a reprodutibilidade dos resultados, todas as medidas foram repetidas pelo menos três vezes. Desta forma, realizou-se um total de 120 testes. Em cada teste foram caracterizadas três situações distintas: a ruptura (primeiro pico de tensão), a falha em movimento (tensão dinâmica estável) e a reativação da falha (segundo pico de tensão). 


\section{Resultados}

A Figura 5 mostra a variação da tensão cisalhante com o tempo, no exemplo da areia azul quando submetida às cinco tensões normais de carregamento (800 Pa, $1200 \mathrm{~Pa}, 1600 \mathrm{~Pa}, 2000 \mathrm{~Pa} \mathrm{e}$ $2400 \mathrm{~Pa}$ ). Percebe-se, em todas as curvas,

Figura 5

Diagrama tensão cisalhante versus tempo obtido no ring-shear tester para a areia azul, sob diferentes tensões normais de carregamento.

então, subir novamente, formando uma curva similar à primeira. Neste ponto, a curva mostra que o aparelho foi desligado e, imediatamente, religado. $\mathrm{O}$ intuito desse procedimento é analisar as condições da tensão cisalhante durante a reativação da falha. As curvas revelam que o segun-

Figura 6

Diagrama tensão cisalhante versus tempo mostrando os resultados obtidos para todas as areias examinadas, sob tensão normal de carregamento de $1600 \mathrm{~Pa}$.

Figura 7

Tensão cisalhante $\left(\sigma_{\mathrm{s}}\right)$ em função da tensão normal $\left(\sigma_{N}\right)$ para todas as areias examinadas. As cinco linhas retas correspondem a envoltórias de Coulomb, que representam o instante do falhamento (o primeiro pico da tensão cisalhante), obtidas por meio de análises de regressão linear. C é a coesão, determinada a partir da extrapolação linear de cada reta. um segmento inicial reto, que representa a fase elástica da deformação da areia. Segue um estágio de strain hardening, plástico, até ocorrer a formação da fratura no primeiro pico da curva, quando a tensão cisalhante é máxima. A deformação con-

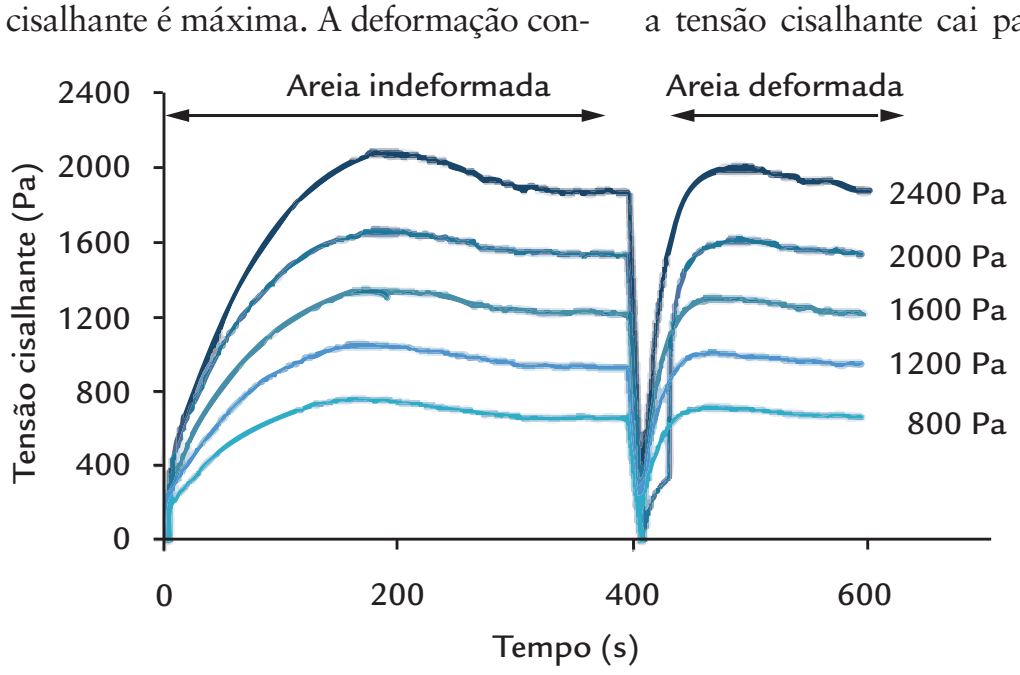

do pico de tensão é menor do que o primeiro, o que corresponde às expectativas, uma vez que a tensão cisalhante necessária para a reativação da falha é menor do que a de sua nucleação (Byerlee, 1978).

Na Figura 6, observa-se a variação da tensão cisalhante com o tempo, sob
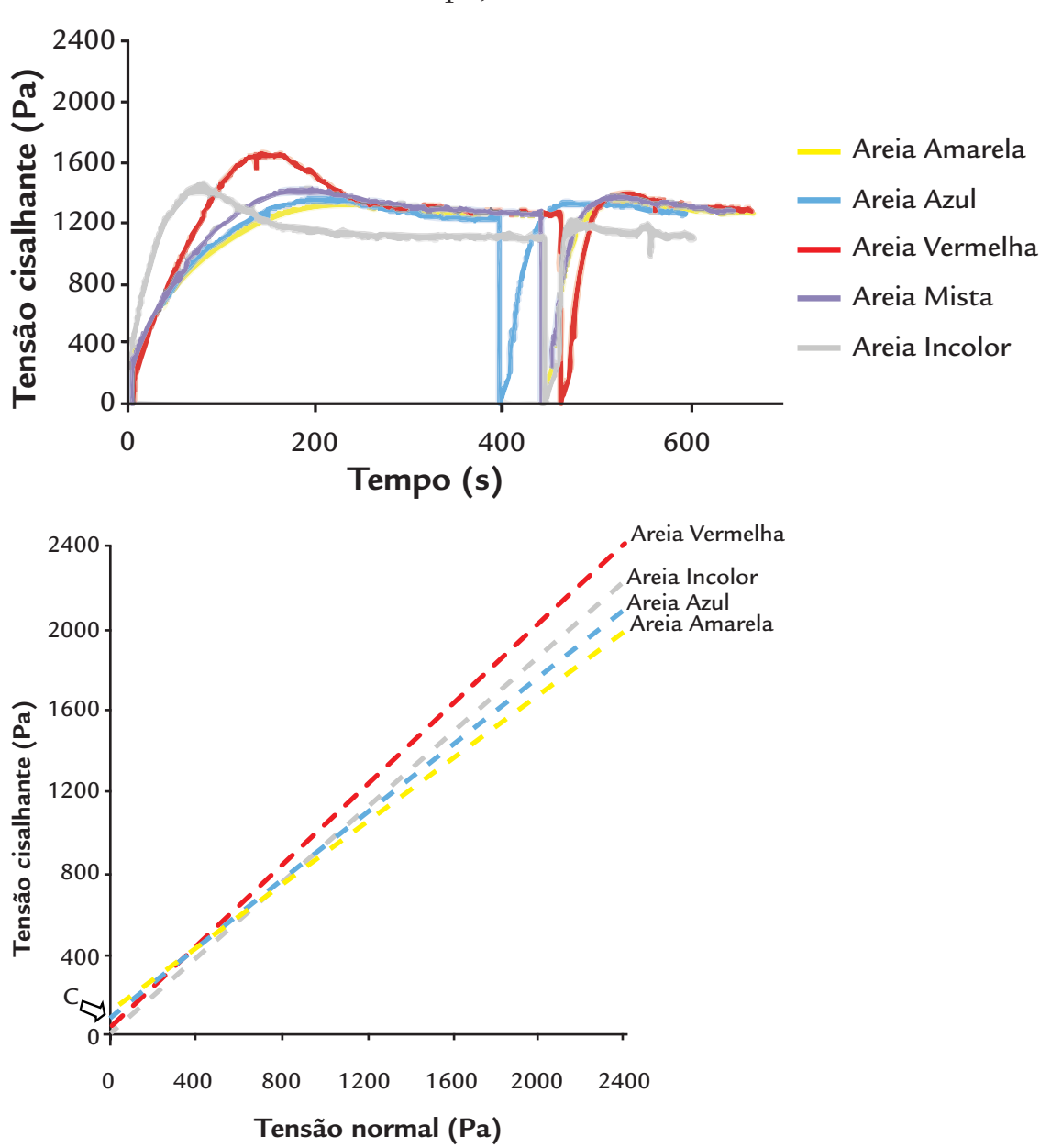

tinua com uma fase de strain-softening até a estabilização da tensão cisalhante - o "estágio da tensão dinâmica estável". Segundo Lohrmann et al. (2003), esta tensão mantém a falha ativa. A seguir, a tensão cisalhante cai para zero, para,

mesma tensão normal de carregamento, de $1600 \mathrm{~Pa}$, para os diferentes tipos de areias examinadas. Percebe-se que a areia mista produziu propriedades friccionais muito próximas daquelas das areias azul e amarela, com pouca influência da areia vermelha. A areia vermelha se diferen- 


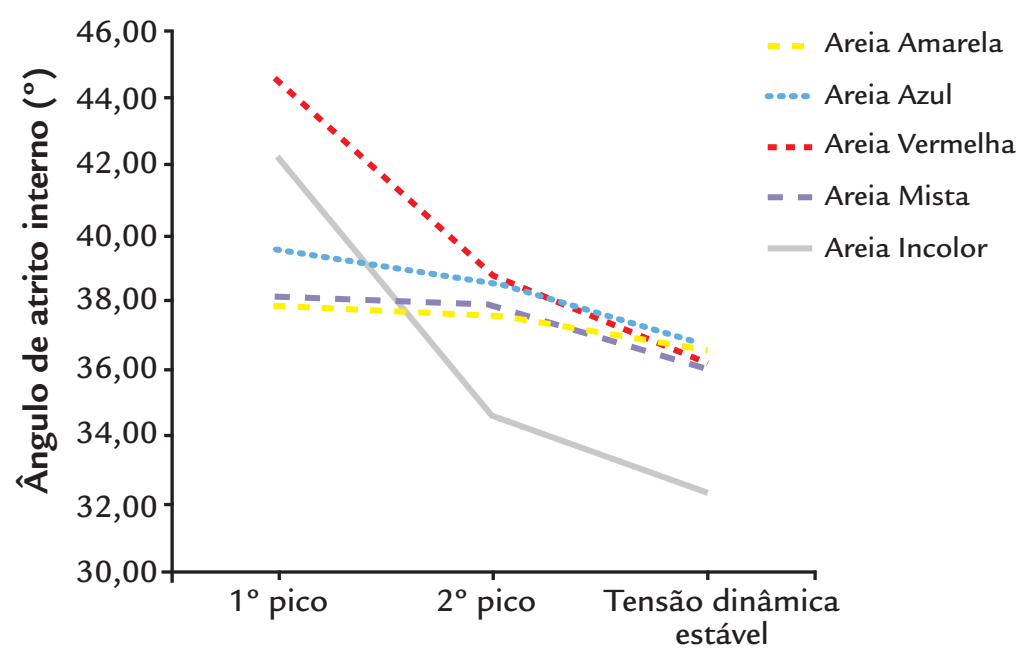

cia da amarela, da azul e da mista pelo primeiro pico de tensão, que é mais alto. Finalmente, a areia incolor mostra uma curva da variação da tensão cisalhante com o tempo, diferente de todas.

É possível que a granulometria ligeiramente mais fina da areia vermelha tenha conduzido ao resultado observado posto que um estudo sobre materiais granulares efetuado por Krantz (1991) demonstrou que a argila quando misturada à areia não modifica de forma significativa o ângulo de atrito interno da areia.

A análise cuidadosa das cinco curvas mostra que a areia incolor se diferencia das demais pela diferença na magnitude do strain softening. Esse parâmetro pode ser calculado pela equação:

\begin{tabular}{c|c|c|c|c|c|c|c|c|c|c|c|c|c|c}
\hline & $\begin{array}{c}\Phi_{\text {primeiro pico }} \\
\left({ }^{\circ}\right)\end{array}$ & $\mu_{\text {primeiro pico }}$ & $\begin{array}{c}C_{\text {primeiro pico }} \\
(\mathrm{Pa})\end{array}$ & $\mathrm{R}^{2}$ & $\begin{array}{c}\Phi_{\text {segundo pico }} \\
\left({ }^{\circ}\right)\end{array}$ & $\mu_{\text {segundo pico }}$ & $\begin{array}{c}C_{\text {segundo pico }} \\
(\mathrm{Pa})\end{array}$ & $\mathrm{R}^{2}$ & $\begin{array}{c}\Phi_{\text {estável }} \\
\left({ }^{\circ}\right)\end{array}$ & $\mu_{\text {estavel }}$ & $C_{\text {estavel }}(\mathrm{Pa})$ & $\mathrm{R}^{2}$ & $\begin{array}{c}\text { Strain } \\
\text { softening } \\
(\%)\end{array}$ & $\begin{array}{c}\rho \\
\left(\mathrm{g} / \mathrm{cm}^{3}\right)\end{array}$ \\
\hline $\begin{array}{c}\text { Areia } \\
\text { azul }\end{array}$ & 39,5 & 0,82 & 108,3 & 0,9761 & 38,6 & 0,80 & 77 & 0,9965 & 36,8 & 0,75 & 65,4 & 0,9968 & 7 & 1,05 \\
\hline $\begin{array}{c}\text { Areia } \\
\text { amarela }\end{array}$ & 37,9 & 0,78 & 117,8 & 0,9960 & 37,6 & 0,77 & 91,7 & 0,9980 & 36,5 & 0,74 & 60,1 & 0,9940 & 4 & 1,05 \\
\hline $\begin{array}{c}\text { Areia } \\
\text { mista }\end{array}$ & 38,1 & 0,78 & 147,9 & 0,9970 & 37,9 & 0,78 & 105,7 & 0,9960 & 36,0 & 0,73 & 87,2 & 0,9960 & 6 & 1,13 \\
\hline $\begin{array}{c}\text { Areia } \\
\text { vermelha }\end{array}$ & 44,5 & 0,98 & 37,7 & 0,9956 & 38,8 & 0,80 & 90,3 & 0,9951 & 36,2 & 0,73 & 78,8 & 0,9947 & 23 & 1,21 \\
\hline $\begin{array}{c}\text { Areia } \\
\text { incolor }\end{array}$ & 42,7 & 0,92 & 36,3 & 0,9969 & 34,6 & 0,69 & 104,7 & 0,9990 & 32,3 & 0,63 & 89,13 & 0,9990 & 32 & 1,52 \\
\hline
\end{tabular}

\section{Discussão}

\section{Comparação entre os dois métodos}

O estudo desenvolvido a partir dos modelos físico-analógicos forneceu valores de ângulo de atrito interno $(\Phi)$ mais baixos do que aqueles obtidos no ringshear tester. As seções centrais dos modelos experimentais "não-compactados" (mais homogêneos) produziram resultados de $\Phi$ entre $29,5^{\circ}$ e $30,0^{\circ}$, e o ringshear tester, entre $37,9^{\circ}$ e $44,5^{\circ}$ (primeiro $\mu_{\text {primeiro pico }}-\mu_{\text {estável }} / \mu_{\text {estável }}$

onde $\mu$ é o coeficiente de atrito interno. $\mathrm{O}$ resultado, expresso em porcentagem, corresponde a $32 \%$ para a areia incolor, $23 \%$ para a areia vermelha e $7 \%, 4 \%$ e $6 \%$ para as areias azul, amarela e mista, respectivamente (Tabela 2 ).

A Figura 7 apresenta as envoltórias de ruptura de Coulomb de todas as areias examinadas (do primeiro pico de tensão). O diagrama mostra que a areia vermelha possui o ângulo de atrito mais elevado, e, a areia amarela, o mais baixo. Este resultado confere com aquele da Figura 6, na qual a tensão cisalhante crítica também decresce da areia vermelha à amarela. Os dados de coesão, do ângulo e do coeficiente de atrito interno de todas pico de tensão cisalhante). Considerando-se, no entanto, todos os valores de $\Phi$ obtidos nos modelos analógicos, que oscilam entre $21,8^{\circ}$ a $39,0^{\circ}$, para os experimentos "não-compactados", e, entre $21,2^{\circ}$ a $36,9^{\circ}$, para os "compactados", observa-se uma ligeira superposição dos dados em relação àqueles obtidos no ring-shear tester.
Figura 8

Ângulo de atrito interno: no primeiro pico de tensão (instante do rompimento), no segundo pico (instante da reativação) e durante a tensão dinâmica estável, para todas as areias examinadas.

as areias examinadas encontram-se reunidos na Tabela 2.

O gráfico da Figura 8 mostra os valores dos ângulos de atrito interno nas três situações analisadas: no instante do primeiro e do segundo picos de tensão e para a tensão dinâmica estável. Da mesma forma como na Figura 6, as areias vermelha e incolor demonstram comportamento anômalo em relação às três outras areias. No instante do fraturamento (o primeiro pico), o ângulo de atrito interno das areias azul, amarela e mista corresponde a $39,5^{\circ}, 37,9^{\circ}$ e $38,1^{\circ}$, respectivamente. A areia vermelha possui ângulo de atrito interno de $44,5^{\circ}$ e a incolor de $42,7^{\circ}$. Para o segundo pico e para a tensão dinâmica estável apenas a areia incolor mostra comportamento diferenciado.

Tabela 2

Valores das propriedades friccionais dos vários tipos de areias examinadas no ring-shear tester ( $\Phi$ - ângulo de atrito interno; $\mu$ - coeficiente de atrito interno; $\mathrm{C}$ - coesão; $\mathrm{R}^{2}$ - quadrado de coeficiente de correlação); taxa de strain softening e densidade aparente $(\rho)$ dos materiais.
A variação dos resultados entre os dois métodos é atribuída a fatores intrínsecos à modelagem física analógica, à forma simplificada da determinação do ângulo de mergulho das falhas de empurrão e à montagem do modelo experimental, uma vez que no ring-shear tester não se trabalha com a areia disposta em camadas.

A pesquisa demonstrou que o ring- 
shear tester produz resultados estatísticos mais precisos do que os modelos experimentais, pois os testes registraram um coeficiente de correlação $\mathrm{R}^{2}$ entre 0,9956 e
0,9970 (primeiro pico de tensão cisalhante) (Tabela 3). Isto significa que os resultados obtidos explicam 99,56 a 99,70\% da variação da tensão cisalhante. Nos experi- mentos físicos, o ângulo de atrito interno (Ф) varia de 44,10\% e de 42,55\%, nos modelos "não compactados" e "compactados", respectivamente.

\section{Comparação entre as propriedades friccionais das areias estudadas, no ring-shear tester}

Na comparação das curvas de tensão cisalhante versus tempo (Figura 6), percebe-se que a areia vermelha possui comportamento intermediário entre a incolor e as areias azul e amarela. $\mathrm{Na}$ areia vermelha, o primeiro pico de tensão cisalhante encontra-se próximo àquele da areia incolor, mas a tensão dinâmica estável e o segundo pico são similares àqueles das areias azul e amarela. O fato sugere que a tinta influencia as propriedades friccionais da areia e que, no caso das tintas Acrilex, aqui empregadas, estas não apresentam composição química homogênea.

É importante destacar que as curvas da variação da tensão cisalhante com o tempo, geradas no presente estudo, se assemelham àquelas obtidas por Ellis et al. (2004) e Panien et al. (2006). No en-

Figura 9

Diagrama tensão cisalhante versus tempo para a comparação do comportamento das areias de marcas diferentes (Acrilex e Xadrez) e incolor. tanto, existe uma pequena diferença no strain softening das curvas das areias azul e amarela. Além disto, às vezes, as curvas obtidas no presente estudo apresentam o segundo pico de tensão cisalhante igual ao primeiro (situação não mostrada nas figuras). Neste caso, a tensão cisalhante necessária à reativação da falha foi igual à de sua nucleação. Esse fato contraria a tese de Byerlee (1978), sugerindo, mais uma vez, que as tintas podem ter alterado as propriedades friccionais da areia natural.

Para efeito de comparação, determinaram-se as propriedades friccionais da areia tingida com a cor verde da marca Xadrez. A Figura 9 revela que essa tinta também muda as propriedades da areia incolor $\left(\Phi=42,92^{\circ} ; \mu=0,93 ; C=81,40 \mathrm{~Pa} ; \mathrm{R}^{2}=\right.$ $0,9964)$. Testes com outras cores da tinta
Xadrez são, no entanto, necessários para se avaliar a magnitude dessas modificações.

Futuros trabalhos deverão ser efetuados para indicar se diferentes cores de outras marcas de tintas também produzem resultados distintos, posto que, no ring-shear tester, a fratura se forma em posição horizontal, afetando um único tipo de areia. Como nos modelos físicos as falhas são inclinadas, de maneira a cortar várias camadas, recomenda-se evitar a condição anteriormente descrita. Com o intuito de se contornar o problema, examinaram-se as propriedades friccionais da mistura das areias azul, amarela e vermelha. O resultado, no entanto, não foi satisfatório, posto que as variações na composição química da tinta Acrilex alteraram a granulometria das areias, de forma diferenciada.

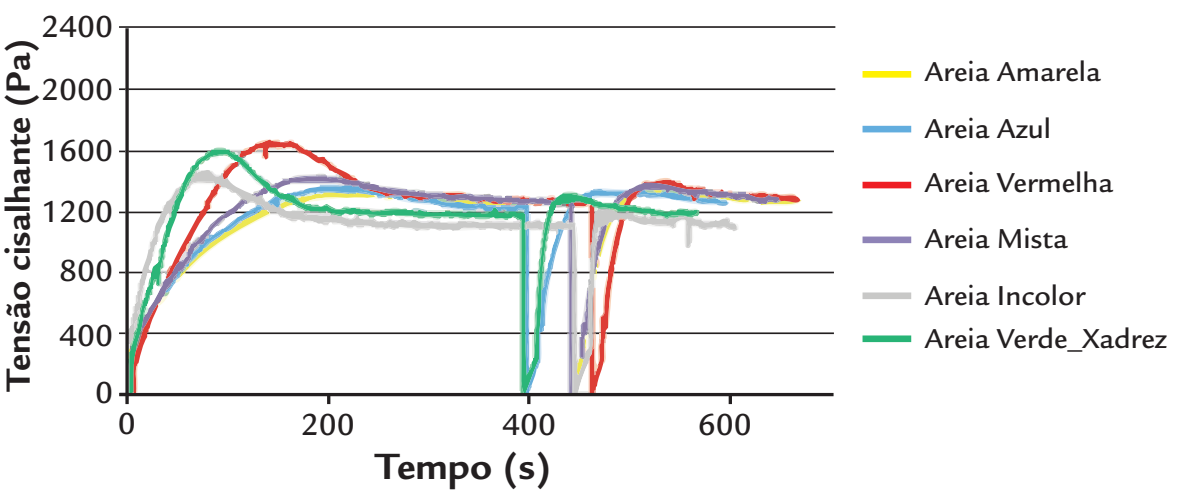

\section{Comparação dos resultados com dados da literatura}

No ano de 1991, Krantz determinou as propriedades friccionais de materiais granulares também a partir de dois métodos: em modelos físicos-analógicos, a partir do ângulo de mergulho de falhas normais, e em um shear-box, do tipo Hubbert (1951). No trabalho do autor, esses dois métodos forneceram resultados similares para o ângulo de atrito interno.

No mesmo estudo, o autor mostra que existe uma relação positiva entre o coeficiente de atrito interno $(\mu)$ e a densidade aparente da areia. Além disto, Krantz (1991) demonstra que a densidade aparente do material granular depende da técnica do manuseio: a areia despejada apresenta densidade aparente pelo menos $15 \%$ abaixo da areia peneirada ou salpicada. Segundo o autor, isto se explica pelo fato de o despejo, mais forte que o do peneiramento, causar um menor grau de encaixe entre os grãos (ou menor número de grãos por volume). Os valores de $\mu$ calculados por Krantz (1991), para a areia despejada, variaram entre 0,40 e 0,58 (igual a $\Phi$ entre $21,8^{\circ}$ e $30,1^{\circ}$ ) e para a areia peneirada, entre 0,93 e 1,04 (igual a $\Phi$ entre $42,9^{\circ}$ e $46,1^{\circ}$ ).

É interessante ressaltar que, no presente trabalho, o processo da leve compactação dos experimentos, durante a montagem, não surtiu efeito similar. Neste processo, se diminuiu, ligeiramente, o volume ocupado pelo pacote de areia, em função do decréscimo do espaço intergranular, porém não associado a um melhor encaixe entre os grãos. As mudanças se restringiram ao encurtamento e, respectivo espessamento do pacote de areia, durante a compressão. Até o aparecimento da primeira falha, em planta, encurtamento e espessamento foram menores no modelo "compactado" do que no "não-compactado" (Figura 10).

Panien et al. (2006) também analisaram o ângulo de atrito interno em um estudo comparativo. Utilizando um CT-Scanner, os autores mediram o ângulo de mergulho de falhas normais, em diversos materiais granulares, e determinaram o ângulo de atrito interno em um ring-shear tester. Esses autores obtiveram ângulos de atrito interno sistematicamente mais elevados para as medidas efetuadas nos modelos experimentais, registrando uma diferença de $16,5^{\circ}$ para a areia de quartzo. A razão para os valores desiguais do ângulo de atrito interno é relacionada às diferenças intrínsicas 


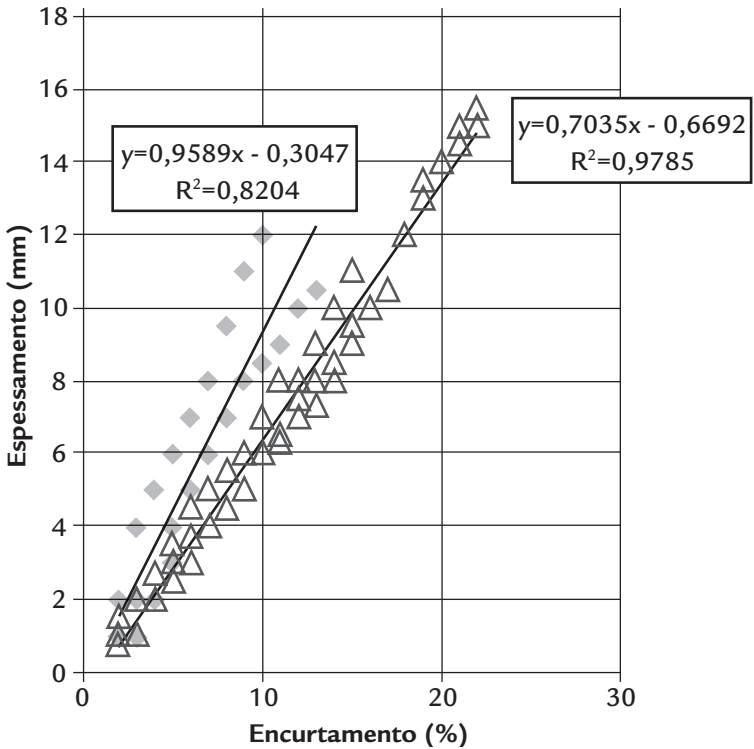

de cada método e ao fato de que, para tensões normais baixas, a envoltória de ruptura constitui, no diagrama de Mohr, uma curva convexa para cima. Para falhas normais, o fato implica valores de coeficiente de atrito interno mais altos para tensões normais baixas. É este o caso dos modelos experimentais, nos quais as tensões normais são mais baixas do que no ring shear-tester.

A Tabela 3 apresenta as propriedades friccionais da areia de quartzo (incolor) de vários autores. Tais autores, apesar de utilizarem diferentes granulometrias e aparelhos distintos, chegaram a conclusões que permitem comparação com os resultados obtidos no presente estudo. Observa-se que o ângulo de atrito interno, $\Phi$, varia entre $29,7^{\circ}$ e $42,0^{\circ}$, o que coloca a areia incolor aqui examinada $\left(42,7^{\circ}\right)$ entre as areias de $\Phi$ mais elevado. É interessante, ainda, notar que o ângulo de atrito interno de $42,7^{\circ}$ difere em $3,4^{\circ}$ do valor 3). Esses autores examinaram a mesma areia, de mesma granulometria, porém utilizando o ring-shear tester - RST-01. pc, do GFZ Potsdam (Alemanha).

Schreurs et al. (2010) descrevem experimentos desenvolvidos por quatorze laboratórios, sob as mesmas condições de contorno e montados com igual mateobtido por Gomes et al. (2009) (Tabela
Figura 10

Gráfico espessamento versus encurtamento para as duas séries de experimentos, "compactada" $(\diamond)$ e "não-compactada" $(\Delta)$, com respectivas linhas e equações de regressão.

rial analógico. Em perfil, as modelagens mostraram uma evolução estrutural muito similar, evidenciando a reprodutibilidade das observações experimentais. No entanto, os resultados não foram idênticos. Os autores relacionam as heterogeneidades entre os experimentos a fatores humanos e a diferenças na temperatura e na umidade dos laboratórios.

Considerando-se que, normalmente, cada laboratório trabalha com o seu próprio material analógico, fica claro que o profundo conhecimento das propriedades friccionais da areia é essencial para adequar o material equivalente às estruturas da crosta terrestre.

Tabela 3

Granulometria e propriedades friccionais $(\mu=$ coeficiente de atrito interno; $\Phi$ = ângulo de atrito interno e $C=$ coesão) da areia de quartzo testada por diferentes autores (modificado de Rossi \& Storti, 2003). A areia incolor usada nesse trabalho é colocada, ao final, para comparação.

\section{Conclusões}

As principais conclusões sobre a análise da areia de quartzo, utilizandose experimentos analógicos e um ringshear tester, são apresentadas a seguir:

$\mathrm{O}$ ângulo de atrito interno $(\Phi) \mathrm{da}$ areia colorida, determinado em caixas de areia, a partir do ângulo de mergulho $(\alpha)$ de falhas de empurrão, mostrou uma forte variação, entre $25,5^{\circ}$ e $34,1^{\circ}$. Os experimentos "compactados" e "nãocompactados" geraram ângulos de atrito interno muito semelhantes.

O ângulo de atrito interno $(\Phi)$ obtido com o ring-shear tester produziu valores mais precisos (com erro $<1 \%$ ) do que os gerados na caixa de areia. Os valores de $\Phi$ foram determinados para três situações distintas: do instante da ruptu- ra (primeiro pico de tensão), da falha em movimento (tensão dinâmica estável) e da reativação da falha (segundo pico de tensão). $\mathrm{O}$ ângulo $\Phi$ da areia incolor, no instante da ruptura, é de $42,7^{\circ}$ e o das areias tingidas de vermelho, azul e amarelo, de $44,5^{\circ}, 39,5^{\circ}$ e $37,9^{\circ}$, respectivamente. Esses valores são considerados altos, mas se enquadram entre os valores 
máximos encontrados na literatura.

As análises no ring-shear tester confirmaram o comportamento elasto/ plástico da areia. Apesar do comportamento anômalo causado por algumas tintas da marca Acrilex, as areias exami-

\section{Agradecimentos}

Os autores desejam expressar os seus agradecimentos à Petrobras pelo fi-

\section{Referências bibliográficas}

nadas mostraram, via de regra, ângulo de atrito interno maior para a nucleação da falha do que para a sua reativação.

Observou-se, ainda, que a tinta Acrilex, usada nos experimentos, para o tingimento da areia, produz alterações nas propriedades friccionais da areia incolor. Além disto, as diferentes cores podem gerar comportamentos distintos. $\mathrm{O}$ fato requer atenção nas futuras modelagens experimentais. nanciamento do projeto que permitiu a aquisição do ring-shear tester e ao CNPq pela bolsa PIBIC.
ACOCELlA, V., FACCENNA, C., FUNICIELlO, R., ROSSETTI, F. Analogue modeling of extensional transfer zones. Bol. Soc. Geol. Ital., v. 119, p. 85-96, 2000.

BONINI, M., SOKOUTIS, D., MULUGETA, G., KATRIVANOS, E. Modeling hanging wall accommodation above rigid thrust ramps. J. Struct. Geol., v. 22, p. 1165-1179, 2000.

BYERLEE, J. Friction of rocks. Pure and Applied Geophysics, v. 116, p. 615-626, 1978.

COBBOLD, P.R., DURAND, S., MOURGUES R. Sandbox modeling of thrust weadges with fluid-assisted detachments. Tectonophys., v. 334, p. 245-258, 2001.

ELLIS, S., SCHREURS, G., PANIEN, M. Comparisons between analogue and numerical models of thrust wedge development. Journal of Structural Geology, v. 26, p. 1659-167, 2004

FACCENNA, C., NALPAS, T., BRUN, J.P., DAVY P. The influence of pre-existing thrust faults on normal fault geometry in nature and experiments. J. Struct. Geol., v. 17, p. 1139-1149, 1995.

GOMES, C. J. S., SIlVA, F. C. A., ROSENAU, M., KLINKMÜLlER, M. As propriedades mecânicas da areia de quartzo medidas em um Ring-Shear Tester. In: SIMP. NAC. ESTUDOS TECTÔNICOS, 12. INTERN. SYMP. TECTONICS, 6, 2009. Ouro Preto. Programa e Resumos: SBG, 2009, p. 47.

GUTSCHER, M., KUKOWSKI, N., MALAVIEILLE, J., LALLEMAND, S. Material transfer in accrecgtionary wedges from analysis of a systematic series of analog experiments. J. Struct. Geol., v. 20, p. 407-416, 1998.

HUBBERT, M. K. Mechanical basis for certain familiar geologic structures. Geological Society of America Bulletin, v. 62, p. 355-372, 1951.

KRANTZ, R. W. Measurements of friction coefficients and cohesion for faulting and fault reactivation in laboratory models using sand and sand mixtures. Tectonophysics, v. 188, p. 203-207, 1991.

LOHRMANN, J., KUKOWSKI, N., ADAM, J., ONCKEN, O. The impact of analogue material properties on the geometry, kinematics and dynamics of convergent sand wedges. Journal of Structural Geology, v. 25, p. 1691-1711, 2003.

MANDL, G. Mechanics of Tectonic Faulting. Models and Basic Concepts. Amsterdam: Elsevier, 1988. 407p.

McCLAY, K. R. Extensional fault systems in sedimentary basins: a review of analogue model studies. Marine and Petrol. Geol., v.7, p. 206- 233, 1990.

PANIEN, M., SCHREURS, G., PFIFFNER, A. Mechanical behaviour of granular materials used in analogue modeling: insights from grain characterization, ringshear tests and analogue experiments. Journal of Structural Geology, v. 28, p. 1710-1724, 2006.

ROSSI, D., STORTI, F. New artificial granular materials for analogue laboratory experiments: aluminium and silicious microspheres. Journal of Structural Geology, v. 25, p. 1893-1899, 2003.

SCHELLART, W.P. Shear test results for cohesion and friction coefficients for different granular materials: scaling implications for their usage in analogue modeling. Tectonophysics, v. 324, p.1-16, 2000.

SCHULZE, D. Entwicklung und Anwendung eines neuartigen Ringschergerätes. Aufbereitungs-Technik, v. 35, n. 10, p. 524-535, 1994.

SCHREURS, G., BUITER, S., BURBERRY, C., CALlOT, J.P., CAVOZZI, C., 
CERCA, M., CRISTALLINI, E., CRUDEN, A., CHEN, J.H., CRUZ, L., DANIEL, J.M., GARCIA, V.H., GOMES, C.J.S., GRALL, C., GUZMÁN, C., HIDAYAH, T.N., HILLEY, G., CHIA-YU LU, KLINKMÜLLER, M., KOYI, M., MACAULEY, J., MAILLOT, B., MERIAUX, C., NILFOUROUSHAN, F., PAN, C.C., PILLOT, D., PORTILLO, R., ROSENAU, M., SCHELLART, W.P., SCHLISCHE, R., TAKE, A., VENDEVILLE, B., VETTORI, M., VERGNAUD, M., WANG, S.H., WITHJACK, M., YAGUPSKY, D., YAMADA, Y. Quantitative comparisons of analogue models of brittle wedge dynamics. In: European Geosciences Union - General Assembly, 2010, Viena, Geophysical Research Abstracts, EGU, 2010, v. 12, p. 11709.

Artigo recebido em 27 de agosto de 2010. Aprovado em 01 de abril de 2011. 